\title{
Ballistic magnetoresistance in a magnetic nanometer sized contact: An effective gate for spintronics
}

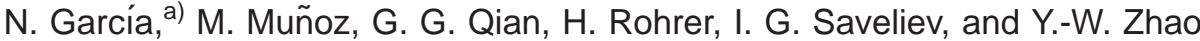 \\ Laboratorio de Física de Sistemas Pequeños y Nanotecnología, Consejo Superior de Investigaciones \\ Cientificas (CSIC), Serrano 144, E-28006 Madrid, Spain
}

(Received 18 September 2001; accepted for publication 10 October 2001)

\begin{abstract}
We present experimental results of unprecedented large magnetoresistance obtained in stable electrodeposited $\mathrm{Ni}-\mathrm{Ni}$ nanocontacts $10-30 \mathrm{~nm}$ in diameter. The contacts exhibit magnetoresistance of up to $700 \%$ at room temperature and low applied fields and, therefore, act as very effective spin filters. These large values of the magnetoresistance are attributed to spin ballistic transport through a magnetic "dead layer" at the contact of width of about $1 \mathrm{~nm}$ or smaller. Nanometer sized, high sensitive magnetoresistive sensors could become key elements for magnetic storage in the terabit/in. ${ }^{2}$ range and in high density magnetic random access memories. (C) 2001 American Institute of Physics. [DOI: 10.1063/1.1427152]
\end{abstract}

One impetus for propelling new technologies is the demand for ever higher bit densities in storage and in memories. In both, the density so far has been doubled every 18 months in recent times. In magnetic storage, it reaches now 10-20 Gbits/in ${ }^{2}$. State-of-the-art reading heads make use of giant magnetoresistance (GMR). ${ }^{1,2}$ The ever increasing storage densities and increasing reading speeds require ever smaller and in particular more sensitive reading heads. For an envisaged density in the range of terabits/in ${ }^{2}$, the bit size shrinks to a few tens of nanometers, thereby demanding reading heads of similar size and of very high sensitivity. The ballistic magnetoresistance (BMR) in magnetic constrictions offers a very promising, new avenue for nanometer scale, ultrasensitive magnetic reading heads. It has been shown that atomic size $(\sim 1 \mathrm{~nm}) \mathrm{Ni}$ and Co contacts exhibit a BMR of up to $300 \%$ at room temperature (RT) and 100 Oe. ${ }^{3}$ This is an order of magnitude larger than what is commonly obtained in GMR. Such large values of magnetoresistance are caused by spin scattering at very thin domain walls. ${ }^{4,5}$ Atomic size contacts, however, were found to be very delicate and are thus not practical for technological applications. Recently we have developed electrodeposited Ni contacts with diameters in the $10-30 \mathrm{~nm}$ range which were stable for days and exhibited BMR of $20 \%$ at RT and 500 Oe. ${ }^{6,7}$ In addition, BMR can be manipulated by current pulses through the contact. ${ }^{6,7}$

The electrodeposited Ni nanocontacts here were grown between two Ni wires of $125 \mu \mathrm{m}$ diameter and $5 \mathrm{~mm}$ length [see Fig. 1(a)], similar to the previous work. ${ }^{6,7}$ However, the contacts were grown at the middle of a horizontal wire and a bipotentiostat (model AFCBP1, Pine Instrument Company) was used in this work to control the potential applied to the two electrodes. Nickel was deposited on the vertical electrode [see Fig. 1(a)] only, so the horizontal electrode remained unaffected by the electrochemical process. The electrolyte was composed of saturated $\mathrm{NiSO}_{4}$ aqueous solution with a $p \mathrm{H}$ value of $1-2$. The deposition potential was chosen

${ }^{\text {a)} C o r r e s p o n d i n g ~ a u t h o r ; ~ e l e c t r o n i c ~ m a i l: ~ n i c o l a s . g a r c i a @ f s p . c s i c . e s ~}$ to be $-1.05 \mathrm{~V}$ versus the saturated calomel electrode (SCE) based on cyclic voltammetry experiments performed in the electrolyte solution employed.

For the magnetoresistance measurement, the sample was placed into a magnetic system (model M-50 MMR Technologies, Inc.) which can provide a homogeneous field up to 4.5 kOe and control the temperature in the range of $80-700 \mathrm{~K}$ with precision of $0.02 \mathrm{~K}$. The magnetic field is applied along the vertical Ni wire axis [see Fig. 1(a)]. The nanocontact resistance, $R$, is measured at currents of $5-100 \mu \mathrm{A}$. We have chosen the crossbar configuration of Fig. 1(a), so that the two sides of the nanocontact respond differently to an applied field along the vertical wire axis due to different shape anisotropies. This different response is the cause of the MR measured. The measured MR values are thus given by the magnetic configuration in the vicinity of the contact which can be induced by a uniform field. They and the associated fields, therefore, can be much smaller or larger, respectively, than the full MR obtained and the fields needed when changing the magnetizations independently on the two sides of the

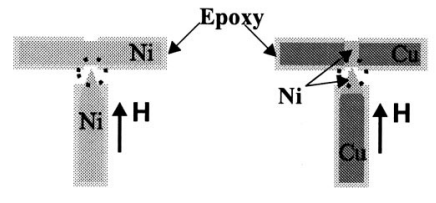

a)

b)

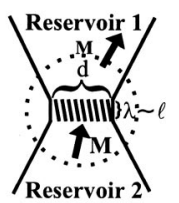

c) $\mathrm{H}=0$

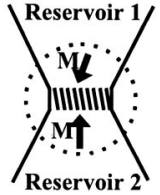

d) H (Rmax)

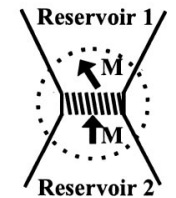

e) $\mathrm{H}(\mathrm{R}<<\mathrm{Rmax})$
FIG. 1. Ni-Ni nanocontacts. Top: Overall configuration: (a) contact on $\mathrm{Ni}$ wires; (b) contact between two very thin Ni layers covering the electrolyte exposed part of the $\mathrm{Cu}$ wires. Bottom: Blowup at the contact. The hatched region represents the domain wall of width $\lambda . \ell$ is the contact length and $d$ its diameter. The arrows indicate possible magnetic configurations of the spin reservoirs adjacent to the contact for the experiment shown in Fig. 2. 

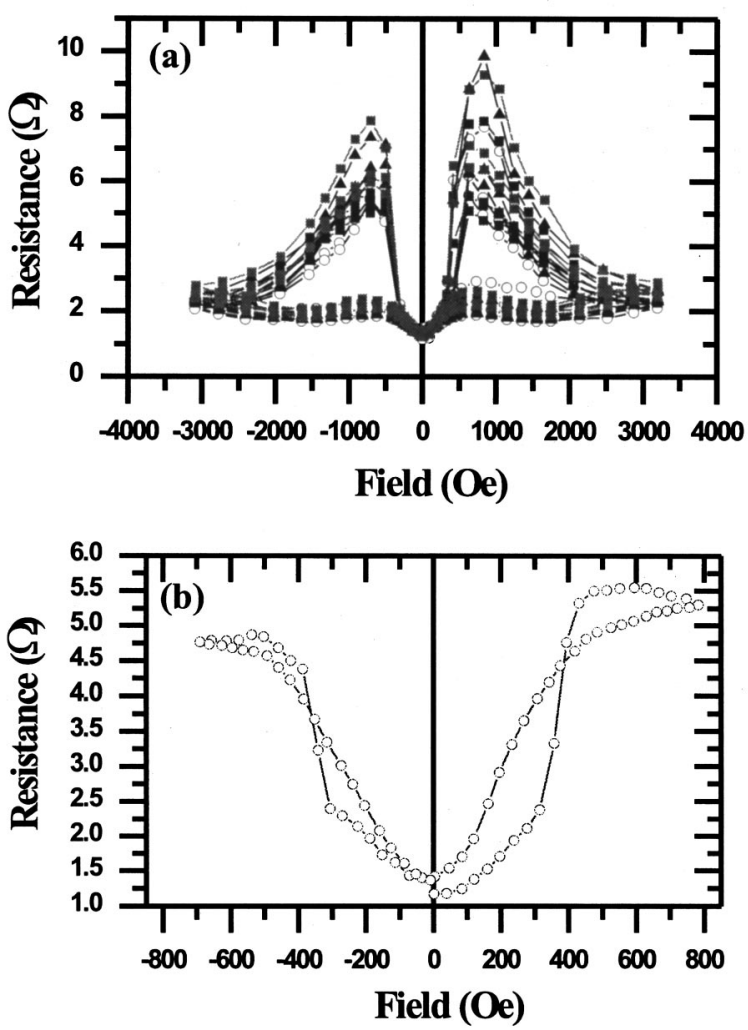

FIG. 2. (a) $13 R(H)$ curves obtained in consecutive field sweeps. The values of the MR decrease with the sweeps and stabilize at a value of about $400 \%$; (b) MR for field cycling between 0 and 700 Oe. The MR is now reversible with the field.

contact from parallel to antiparallel. The resistance is usually lowest in the virgin zero field state, suggesting a predominantly parallel configuration as shown in Fig. 1(c).

In Fig. 2, we present a striking experimental result for a sample of $1.2 \Omega$ initial resistance $(H=0)$, corresponding to a contact diameter of $30 \mathrm{~nm}$. The section size of the nanocontact determines the resistance $R$ : the diameter of the contact, $d$, in $\mathrm{nm}$ is approximately $\left[10^{3} / R(\Omega)\right]^{1 / 2}$, assuming that the quantum of resistance of $12.9 \mathrm{k} \Omega$ is associated with one atom with an area of approximately $0.1 \mathrm{~nm}^{2}$. Figure 2(a) shows 13 full $R(H)$ sweeps from zero field to $3.4 \mathrm{kOe}$ on to $-3.4 \mathrm{kOe}$ and back to zero field, taken within $6 \mathrm{~h}$. The first curve shows the largest magnetoresistance of $700 \%$ in a field of $H=720 \mathrm{Oe}$. The MR goes through a maximum at around $700 \mathrm{Oe}$, before dropping to a small value in high fields. Upon cycling, the MR decreases and tends to stabilize at $\sim 400 \%$, with the field of maximal MR remaining at around 720 Oe. The large hysteresis is attributed to domain wall pinning. If the fields are swept to the MR maximum of 720 Oe only, the hysteresis becomes very small [see Fig. 2(b)]. Since the resistance in zero field is lowest in most of the contacts investigated, up to some small hysteresis effects, the magnetization directions on either side of the contact can be taken as predominantly parallel in a zero applied field. The increase of the MR with applied field means a change to a less parallel magnetic configuration. Actually, as elaborated on below, the maximum in the MR is associated with a predominantly antiparallel configuration. A plausible cause for this behavior is that domains or domain walls sweep across the contact. $^{7,8}$ reported here are conceivable using materials other than $\mathrm{Ni}$
contact.,
Downloaded 15 Feb 2010 to 161.111.180.191. Redistribution subject to AlP license or copyright; see http://apl.aip.org/apl/copyright.jsp
Therefore, the micromagnetic configuration in the vicinity of the contact, which determines the magnetoresistance, is not connected in a straightforward, unambiguous way to the external field and thus to the bulk magnetization. On the one hand, this is a drawback inherent to the present experimental setup. On the other hand, it demonstrates that the measured MR is not an artifact due to magnetostatic forces and magnetostriction, since these are given by the bulk magnetic properties and not by the micromagnetic configurations in the vicinity of the contact. This is further corroborated by experiments, in which the $\mathrm{Ni}$ wires were replaced by $\mathrm{Cu}$ wires on which the $\mathrm{Ni}-\mathrm{Ni}$ contact was grown after a $\mathrm{Ni}$ layer of a few $\mu \mathrm{m}$ thickness and $1 \mathrm{~mm}$ length was first deposited on the center of the horizontal $\mathrm{Cu}$ wire and on the tip of the vertical $\mathrm{Cu}$ wire [see Fig. 1(b)].

We attribute the decrease of the maximal MR in Fig. 2 of $700 \%$ to about $400 \%$ to a long relaxation time of the micromagnetic configuration in the vicinity of the contact. We, however, exclude oxidation of the contact as a possible cause. First, oxidation should critically affect the zero field resistance, which, however, remains unchanged. Furthermore, a very thin electron transparent oxide layer with conducting channels, defects, etc., is not expected to reduce the BMR. Second, a similar decrease of the MR is also observed in contacts which are kept in the electrolyte, where no oxidation is expected.

These extraordinary large values of the MR are a consequence of ballistic conductance. The theory to explain BMR is based on the strong scattering suffered by the spin by a thin domain wall. ${ }^{4,5}$ The relevant formula developed in Ref. 5 is

$$
\operatorname{BMR}(\%)=\frac{2 p^{2}}{1-p^{2}} F \times 100,
$$

where $p=\left(D_{\uparrow}-D \downarrow\right) /\left(D_{\uparrow}+D_{\downarrow}\right)$ describes the spin polarization, $D_{\uparrow}$ and $D_{\downarrow}$ are the densities of states for up and down spins at the Fermi level, and $F$ is a function that describes the nonconservation of conduction electron spin. Theory further predicts, ${ }^{4,5}$ that, at a domain wall width comparable to or smaller than the electron wavelength, the spin should be conserved $(F=1)$ in the conduction process and the BMR achievable is given by the degree of polarization only. Assuming that the band structure on the two sides of the nanocontact is the same as that in the bulk electrode, then, for $\mathrm{Ni}$ $D_{\uparrow} / D_{\downarrow}=11,{ }^{9}$ i.e., $p=0.83$. Using Eq. (1) one arrives at a maximal MR of $400 \%$, in good agreement with the experiments. The even larger experimental BMR values, although the experiments should give a lower bound, might be attributed to larger surface electronic polarization than that in the bulk due to a special configuration of atomic positions near the contact region that may be metastable.

From domain wall theory at nanocontacts we know ${ }^{10}$ that the domain wall width $\lambda \sim d$ if $d$ is smaller than the bulk domain wall. Then from scattering from domain wall ${ }^{4,5}$ for $d \sim 10-30 \mathrm{~nm}$ the MR values should be negligible. Therefore we proposed ${ }^{11}$ that a thin magnetic "dead layer" of $\sim 1 \mathrm{~nm}$ or smaller may be created during the electrodeposition process. This layer may conserve spin current $(F=1)$ and provide very large BMR values. Higher BMR values than those 
with higher electron polarization at the Fermi level or by choosing electron transfer between appropriate bands. For the latter, we cite experiments in which fully spin-polarized electrons are injected at $5 \mathrm{eV}$ in UHV into Co monolayers, ${ }^{12}$ resulting in a MR of about $1000 \%$.

In conclusion, we have shown that $10-30 \mathrm{~nm}$ sized electrodeposited nanocontacts exhibit MR values up to $700 \%$ at room temperature and low fields and act as nearly perfect spin filters. The method of fabricating the contacts is straightforward and should lend itself to large scale fabrication. Further development of these kinds of contacts should lead to nanometer sized magnetic sensors with the sensitivity and stability necessary for reading heads using terabit/in. ${ }^{2}$ magnetic storage or for newly proposed nonvolatile magnetic random access memories.

This work was supported by EU Contract No. IST 200026011.

${ }^{1}$ M. N. Baibich, J. M. Broto, A. Fert, F. Nguyen Van Dau, F. Petroff, P. Etienne, G. Creuzet, A. Friederich, and J. Chazelas, Phys. Rev. Lett. 61,
2472 (1988); G. Binasch, P. Grünberg, P. Saurenbach, and W. Zinn, Phys. Rev. B 39, 4828 (1989).

${ }^{2}$ A. E. Berkowitz, J. R. Mitchell, M. J. Carey, A. P. Young, S. Zhang, P. E. Spada, F. T. Parker, A. Hutten, and G. Thomas, Phys. Rev. Lett. 68, 3745 (1992); J. Q. Xiao, J. S. Jiang, and C. L. Chien, ibid. 68, 3749 (1992).

${ }^{3}$ N. García, M. Muñoz, and Y.-W. Zhao, Phys. Rev. Lett. 82, 2923 (1999).

${ }^{4}$ G. G. Cabrera and L. M. Falicov, Phys. Status Solidi B 61, 539 (1974).

${ }^{5}$ G. Tatara, Y.-W. Zhao, M. Muñoz, and N. García, Phys. Rev. Lett. 83, 2030 (1999).

${ }^{6}$ N. García, I. G. Saveliev, Y.-W. Zhao, and A. Zlatkine, J. Magn. Magn. Mater. 214, 7 (2000).

${ }^{7}$ N. García, H. Rohrer, I. G. Saveliev, and Y.-W. Zhao, Phys. Rev. Lett. 85, 3053 (2000).

${ }^{8}$ S. A. Minyukov and N. García, J. Magn. Magn. Mater. 214, 327 (2000).

${ }^{9}$ J. L. Moruzzi, J. F. Janak, and A. R. Williams, Calculated Band Structure Properties (Pergamon, New York, 1978).

${ }^{10}$ P. Bruno, Phys. Rev. Lett. 83, 2425 (1999); N. Garcia, V. V. Osipov, and E. V. Ponizovskaya, Phys. Rev. B (to be published). This paper shows that the domain wall width of a constriction is larger than its diameter if the latter is smaller that the bulk domain wall width.

${ }^{11}$ N. Garcia, M. Muñoz, I. G. Savelier, and Y.-W. Zhao, MML '01 Conference, Aachen, Germany, June 2001.

${ }^{12}$ D. Oberli, R. Burgermeister, S. Riesen, W. Weber, and H. C. Siegmann, Phys. Rev. Lett. 81, 4228 (1998). 